\title{
Enhancement of mechanical properties of an epoxy composite reinforced with Hibiscuss sabdariffa var. altissima fiber micro cellulose
}

\author{
Abhilash Karakoti, A. Soundhar, M. Rajesh, K. Jayakrishna, Mohamed Thariq Bin Haji Hameed \\ Sultan
}

\begin{abstract}
The effects of micro cellulose reinforcement on the mechanical properties of epoxy composites were investigated. Micro cellulose were extracted from Hibiscuss sabdariffa fibers using the steam explosion technique and repeated chemical treatments. Reinforcing of epoxy resin with micro cellulosic fibers was done in particle form. Tensile, hardness and impact results revealed that mechanical properties of micro cellulose reinforced composites increases for 10\%, 20\% and 30\% fiber loading and then decrease for $40 \%$ loading. Composite with $30 \%$ of micro cellulose enhanced the overall mechanical properties of composite, due to the enhanced fiber-matrix adhesion and micro cellulose reinforcement.
\end{abstract}

Index Terms: Cellulose, Composites, Hibiscus sabdariffa var altissima, Hardness.

\section{INTRODUCTION}

Bio-based composites have not only benefitted the eco-system, but also added value to the economic development of farming and rural industries. Natural fibers have been used since the ancient ages where bamboos were used for construction purposes. Over the years, their applications as a bio-filler or reinforcing agent has increased due to their large availability as a waste material from agricultural farms and paper industries $[1,2]$. The plant based cellulosic fibers can be obtained from raw fibers or agricultural wastes such as coffee husk, straw residues, etc. having a larger surface to volume ratio and provides better mechanical properties when compared to raw fibers as a reinforcement [3].

Amount of cellulose in natural fibers defines the strength of the composite material. Researchers used different techniques to extract the cellulose from natural fibers such as steam explosion, chemical treatments or electro-spinning, which will provide large surface to volume ratio and uniform dispersion when mixed in resin [4]. Reinforcement of

Revised Manuscript Received on July 05, 2019.

Abhilash Karakoti, Vellore Institute of Technology , Tamil Nadu -632014, India

A. Soundhar, Vellore Institute of Technology, Tamil Nadu -632014, India M. Rajesh, Vellore Institute of Technology, Tamil Nadu -632014, India K. Jayakrishna*, Vellore Institute of Technology , Tamil Nadu -632014, India. Corresponding author.Email: mail2jaikrish@gmail.com

Assoc. Prof. Ir. Ts. Dr. Mohamed Thariq Hameed Sultan, Department of Aerospace Engineering, Faculty of Engineering, and Institute of Tropical Forestry and Forest Products (INTROP), Universiti Putra Malaysia, 43400 UPM Serdang, Selangor Darul Ehsan, Malaysia and Aerospace Malaysia Innovation Centre (944751-A), Prime Minister's Department, MIGHT Partnership Hub, Jalan Impact, 63000 Cyberjaya, Selangor Darul Ehsan, Malaysia. Corresponding author.Email: thariq@upm.edu.my cellulosic fibers in polymer matrix would give better mechanical properties when compared to short or long fibers reinforced composites because of larger surface to volume ratio and uniform dispersion.

Sonia and Dasan [5] evaluated the effect of celluloses microfiber (CMF) reinforcement in ethylene-vinyl acetate by using xylene, toluene and benzene as penetrant molecule. Cherian et al. [6] examined the effect of steam explosion which caused fibrillation of cellulose pineapple leaf fibers and reinforced the nano fibrils in the polyurethane matrix in order to reveal its potential in medical implants. Bisanda and Ansell [7] found that mercerized and silane treated fibers not only effectively enhance the compressive strength, but also reduces the moisture absorption of the composites. Almari and Low [8] investigated that recycled cellulose fibers improves the mechanical properties because of the reduction in matrix material and availability of fibers to restrain the matrix. Aseer and Sankarnarayanasamy [9] studied the effect of fiber content on tensile retention properties of cellulose microfibers composites and also investigated the degradation behavior of Hibiscus sabdariffa/Polyester by compression molding. Priya et al. [10] described the preparation of cellulosic Grewia optiva fiber reinforced composite cast films from blends of cornstarch and polyvinyl alcohol. Luo et al. [11] also suggested, if steam exposed palm fibers are used for the manufacturing windmill panel they will possess high strength and high toughness. Thakur et al. [12] demonstrated the recent developments and emerging applications of natural cellulose fibers and their polymer composites. Jawaid and Khalil [3] presented an outline on the recent development and potential of cellulosic fibers used in hybrid composites with synthetic fibers for various applications like linings, door panels or fittings.

The literature survey revealed very few available data for the effective utilization of cellulosic fibers as a reinforcing agent. Successful extraction of Hibiscus sabdariffa (HS) cellulosic fibers is already performed by Karakoti et al. [13]. In their study, steam explosion and repeated chemical treatments developed the rough surfaces and reaction sites on the surface of the fiber which will enhance the fiber-matrix bonding resulting in better mechanical properties. In this work cellulosic fibers are reinforced in epoxy matrix with varying fiber ratios of 10, 20, 30 and $40 \mathrm{wt} \%$ of HS fiber. Impact, hardness and tensile tests were conducted for HS fibers reinforced composites and compared with the neat epoxy specimen.

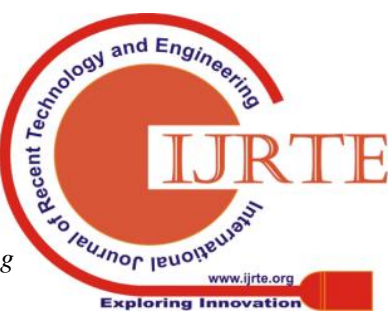




\section{MATERIALS AND METHODOLOGY}

\section{A. Materials}

In this work, HS fiber and epoxy were used as reinforcement and matrix respectively. Reinforcement and matrix (Araldite CY-230 and Hardener HY-951) materials were purchased from local farms of Kolkata and M/s CIBATUL LTD, Delhi respectively. $\mathrm{NaOH}$ and $\mathrm{NaClO}_{2}$ chemicals were procured from SRL chemicals, Delhi and used for extracting the cellulose from HS raw fibers.

\section{B. Composite Fabrication}

Composite laminates used in the study were fabricated using hand-lay-up technique. Initially, micro-cellulose were extracted from HS fiber using the steam explosion technique. Raw fibers were chopped into $10 \mathrm{~cm}$ length and kept at $50^{\circ} \mathrm{C}$ temperature for two hours in the hot air oven to remove the moisture from the fiber. In continuation, chopped fibers were dipped in $\mathrm{NaOH}$ solution at room temperature. After alkali treatment fibers were washed using distilled water thoroughly, followed by steam explosion. Fibers obtained from the steam explosion were soaked in a $\mathrm{NaClO}_{2}$ solution for one hour at $50^{\circ} \mathrm{C}$ in hot air oven. After that, fibers were washed using distilled water and allowed to steam explode for three hours at an approximate temperature and pressure of $120^{\circ} \mathrm{C}$ and 15 psi respectively in an autoclave. The fibers were washed thoroughly with distilled water until neutrality.

Micro-cellulose obtained from the above process, was mixed with high density epoxy matrix and mechanically stirred for two hours to achieve homogenous dispersion. Further the mixture was poured into the mold cavity and closed using a wooden parallel plate. In continuation, composite samples were sized according to ASTM standards ASTM D638-IV and D256 for tensile and impact testing, respectively. Composition of prepared composites is shown in Table 1.

Table 1. Compositions of epoxy filled with H. sabdariffa var. altissima micro fibers

\begin{tabular}{cccc}
\hline $\begin{array}{c}\text { Designation } \\
\text { of } \\
\text { Composition }\end{array}$ & $\begin{array}{c}\text { Epoxy } \\
\text { Resin } \\
(\mathbf{C Y - 2 3 0}) \\
(\mathbf{g m})\end{array}$ & $\begin{array}{c}\text { Hardener } \\
\text { (HY-951) } \\
\text { (gm) }\end{array}$ & $\begin{array}{c}\text { HS fibers } \\
\text { (gm) }\end{array}$ \\
\hline $\begin{array}{c}\text { Neat Epoxy } \\
\text { (NE) }\end{array}$ & 120 & 12 & 0 \\
$\mathbf{1 0 H S}$ & 107 & 11.8 & 13.2 \\
$\mathbf{2 0 H S}$ & 95.04 & 10.56 & 26.4 \\
$\mathbf{3 0 H S}$ & 83.16 & 9.24 & 39.6 \\
$\mathbf{4 0 H S}$ & 71.28 & 7.92 & 52.8 \\
\hline
\end{tabular}

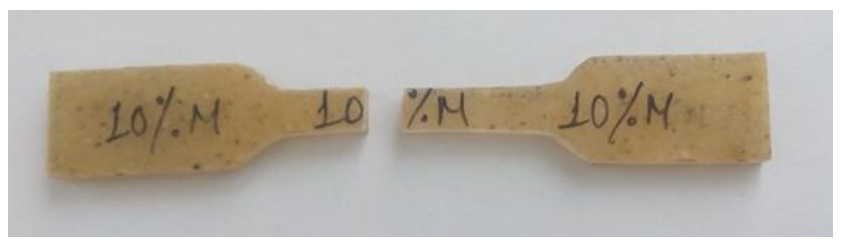

Fig. 1: Tensile tested specimen (ASTM D 638, Type IV).

\section{RESULTS AND DISCUSSIONS}

Fig.1 shows the actual tensile specimen and the effect of micro cellulose reinforcement on the mechanical properties of epoxy composites are shown in Fig. 2. It shows that, HS fiber reinforced composites shows improvement in tensile properties due to the enhancement of interaction between micro cellulose and epoxy which inturn increases the load bearing capacity of the composites. However, it is not significant for higher wt $\%$ of micro cellulose reinforcement. The interfacial strength helps in transfer of load between fiber and matrix. Interfacial strength also depends on the surface morphology of the fiber. Wetting of fibers by epoxy resin and chemical bonding between fibers and epoxy result in better interfacial strength. Thus, tensile properties increases upto 30 wt $\%$ and decreases at the fiber loading of $40 \mathrm{wt} \%$. The main reason behind poor tensile properties for $40 \mathrm{wt} \%$ of micro-cellulose is the agglomeration of cellulose decreases the load bearing capability of the composites under loading. At low levels of fiber wt $\%$, the fibers are not capable of transferring the load to one another, and stress accumulated at certain points of the composite, which led to lower mechanical properties [14]. At the $30 \%$ level of fiber loading, the quantity of the fibers has been just right for active participation of fibers in load transformation hence giving better result.

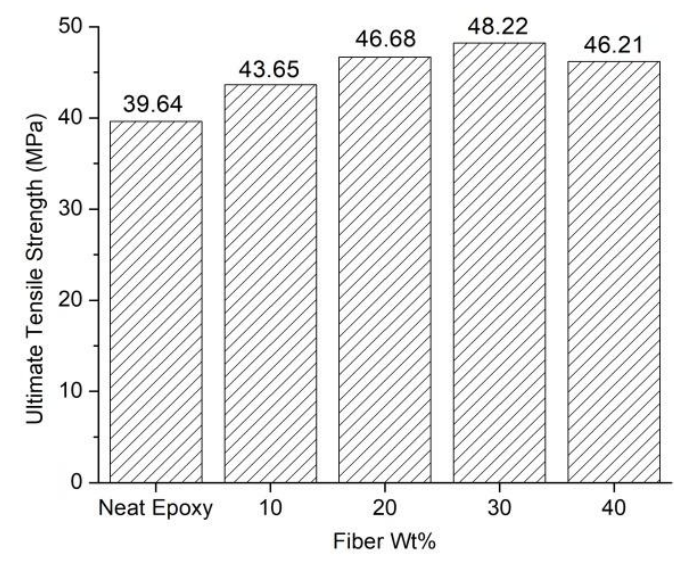

Fig. 2: Ultimate tensile strength of HS composites with varying fiber $\mathrm{wt} \%$

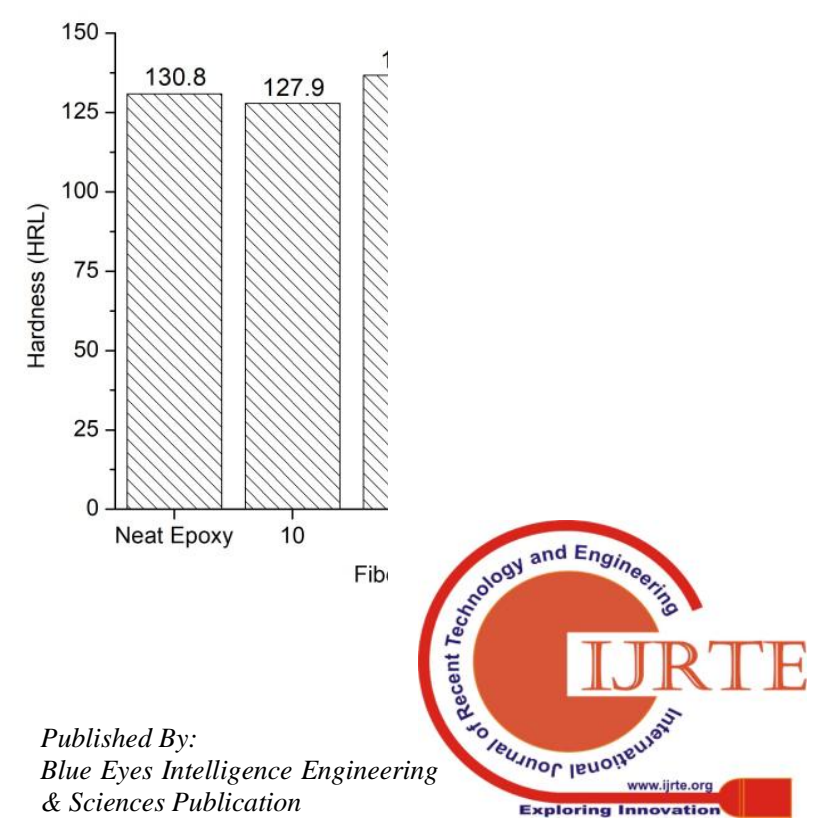


Fig. 3: Hardness of HS composites with varying fiber wt\%.

Fig. 3 revealed the addition of micro cellulose fiber enhanced the hardness property of composite with lower and higher cellulose content. The impact testing specimen is shown in Fig. 4. As shown in Figs. 5 and 6, similar trend was observed where the impact properties increases up to $30 \mathrm{wt} \%$, and decreases at the fiber loading of $40 \mathrm{wt} \%$. The main reason behind higher hardness and impact properties of $30 \mathrm{wt} \%$ is, the strong hydroxyl group present in the celluloses creates link with hydrophobic nature of epoxy matrix which increases the adhesion between fiber and matrix. Hence, it enhanced the load tearing capacity of the composites. However, it is predominant in lower and higher wt $\%$ composites. It is because of the insufficient amount, reinforcement offer a poor load carrying capacity of composites. Same time at higher weight $\%$, composite fails due to the agglomeration effect as it increases the stress concentration under loading [15].

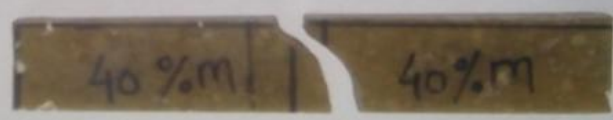

Fig. 4: Impact tested specimen (ASTM D 256).

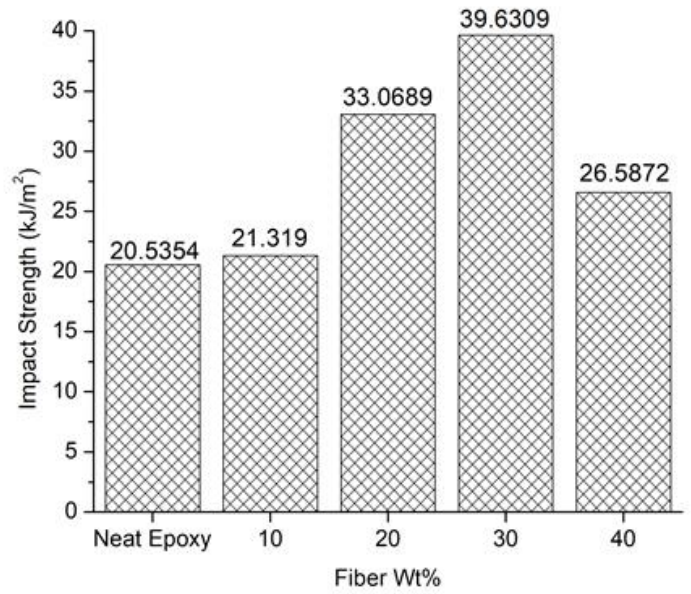

Fig. 5: Impact Strength of HS composites with varying fiber wt $\%$.

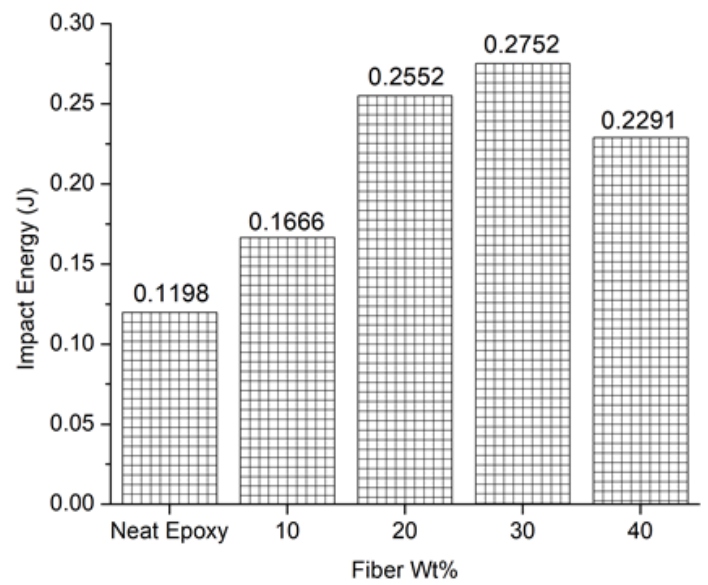
wt $\%$.

\section{CONCLUSIONS}

Epoxy composites reinforced with micro-cellulose extracted from HS fiber have been analysed for mechanical properties such as tensile, hardness and impact. Results revealed that mechanical properties of epoxy composites are influenced by different weight percentage of cellulose. It is found that $30 \mathrm{wt} \%$ micro-cellulose enhances the tensile, hardness and impact strength of the composites by $17.74 \%$, $9.35 \%$ and $48.195 \%$ respectively compared to neat resin. Results revealed that $40 \mathrm{wt} \%$ of micro cellulose reinforcement decreases the mechanical properties of composites due to agglomeration effect.

\section{ACKNOWLEDGMENT}

The authors thank the Universiti Putra Malaysia (UPM), particularly the Department of Aerospace Engineering and Department of Mechanical Engineering for the infrastructures lend, enabling the project to be completed. Not to forget, supervisor of research project, $\mathrm{Dr}$ Norkhairunnisa for invaluable guidance and funding throughout the research project.

\section{REFERENCES}

1. V.P. Arthanarieswaran, A. Kumaravel and S.S. Saravanakumar (2015), Characterization of New Natural Cellulosic Fiber from Acacia leucophloea Bark, International Journal of Polymer Analysis and Characterization 20, pp. 367-376.

2. J. Pitchaimani and M. Rajesh (2018), Dynamic Mechanical and Free Vibration Behavior of Natural Fiber Braided Fabric Composite: Comparison with Conventional and Knitted Fabric Composites, Polymer Composites 39, pp. 2479-2489.

3. M. Jawaid and H.P.S. Abdul Khalil (2011), Cellulosic/synthetic fibre reinforced polymer hybrid composites: A review, Carbohydrate Polymer 86, pp. 1-18.

4. A. Bledzki and J. Gassan (1999), Composites reinforced with cellulose based fibres, Prog. Polym. Sci. 24, pp. 221-274.

5. A. Sonia and K.P. Dasan (2014), Barrier properties of celluloses microfibers (CMF)/ethylene-co-vinyl acetate (EVA)/composites. Composite Interfaces 21, pp. 233-250.

6. B.M. Cherian, A.L. Leão, S.F. de Souza, S. Thomas, L.A. Pothan \& M Kottaisamy (2010), Isolation of nanocellulose from pineapple leaf fibres by steam explosion. Carbohydrate Polymers 81, pp. 720-725.

7. E.T.N. Bisanda and M.P. Ansell (1991), The effect of silane treatmen on the mechanical and physical properties of sisal-epoxy composites. Composites Science and Technology 41, pp. 165-178.

8. H. Alamri and I.M. Low (2012), Mechanical properties and water absorption behaviour of recycled cellulose fibre reinforced epoxy composites. Polymer Testing 31, pp. 620-628.

9. K.Snkarnarayansamy and J.R. Aseer (2017), Effect of fiber content on tensile retention properties of Cellulose Microfiber Reinforced Polymer Composites for Automobile Application. IOP Conf. Series: Materials Science and Engineering 272 , pp. 012020.

10. B. Priya, V.K. Gupta and D. Pathania (2014), A.S. Singha, Synthesis, characterization and antibacterial activity of biodegradable starch/PVA composite films reinforced with cellulosic fibre. Carbohydrate Polymer 109, pp. 171-179.

11. H. Luo, H. Zhang, L. Yue, A. Pizzi and X. Lu (2018), Effects of steam explosion on the characteristics of windmill palm fiber and its application to fiberboard. European Journal of Wood and Wood Product 76, pp. 601-609.

12. V.K. Thakur and M.K. Thakur

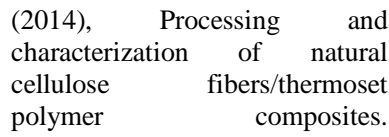

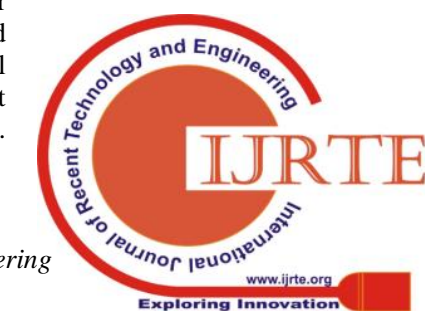


Carbohydrate Polymers 109, pp. 102-117.

13. A. Karakoti, S. Biswas, J.R. Aseer, N. Sindhu and M.R. Sanjay (2018), Characterization of microfiber isolated from Hibiscus sabdariffa var. altissima fiber by steam explosion. Journal of Natural Fibers 0, pp. $1-10$.

14. A.S. Singha and V.K. Thakur (2009), Mechanical, Thermal and Morphological Properties of Grewia Optiva Fiber / Polymer Matrix Composites, Polymer-Plastics Technology and Engineering Mechanical 48, pp. 201-208.

15. A.S. Singha and V.K. Thakur (2008), Synthesis and characterization of Grewia optiva fiber-reinforced PF-based composites, International Journal of Polymeric Materials and Polymeric Biomaterials 57, pp. 1059-1074.

\section{AUTHORS PROFILE}

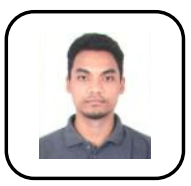

Abhilash Karakoti is a $\mathrm{PhD}$ scholar from School of Mechanical Engineering, Vellore Institute of Technology, majoring in numerical modelling and experimentation of composite materials. He has published two SCI journals, two book chapters and presented five conference papers in international conferences.

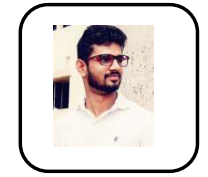

A. Soundhar is a PhD scholar from School of Mechanical Engineering, Vellore Institute of Technology, majoring in natural fiber reinforced bio-composites. He has published two SCI journals, one scopus journal and two book chapters.

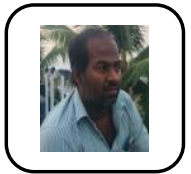

M. Rajesh is a Professor from School of Mechanical Engineering, Vellore Institute of Technology. His area of expertise is natural fiber reinforced composites. To date, he has published more than 27 articles in numerous journals, book chapters, proceedings, etc.

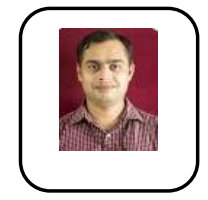

K. Jayakrishna is an Associate Professor in the Schoo of Mechanical Engineering at the Vellore Institute of Technology University, India. His research is focused on sustainable product development. To date, he has published more than 80 articles in numerous journals, book chapters, proceedings, etc.

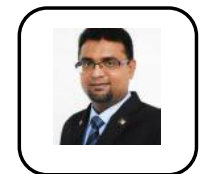

Mohamed Thariq Bin Haji Hameed Sultan is a Professor in Department of Aerospace Engineering, Universiti Putra Malaysia. His area of expertise are Structural Health Monitoring (SHM), damage detections and repairs, impact studies, etc. To date, he has published more than 190 articles in numerous journals, book chapters, proceedings, etc. 